\title{
Emergency Treatment and Nursing Care of 148 Multiple Trauma Patients Complicated with Severe Thoracic Trauma
}

\author{
Wenwen Liu \\ Nursing Department, People's Hospital of Zhengzhou, Zhengzhou 450053, China
}

Keywords: severe thoracic trauma; multiple trauma; emergency treatment and nursing care.

\begin{abstract}
Objective: To discuss the emergency treatment and nursing care of patients with severe thoracic trauma, and learn the lessons to improve the quality of nursing. Methods: Rescue and nursing experience of 148 multiple trauma patients complicated with severe thoracic injury were summarized retrospectively. Results: Among the 148 cases, 138 cases were successfully treated and 10 cases died unfortunately, among which 83 cases were received multidisciplinary joint surgery and 6 cases died. Conclusion: The key of a successful nursing care are to keep the airways open, to maintain the partial pressure of blood oxygen, to stop bleeding actively and anti-shock, to observe nearly the change of illness, to transfer reasonably, to organize rescuer effectively, to cooperate with surgery especially multidisciplinary joint surgery.
\end{abstract}

\section{Introduction}

542 cases with multiple injuries were received and cured by our hospital since 2009-2013, among which 148 cases were severe thoracic trauma patients (AIS $\geq 3$ ). The most common cause of injury was traffic accidents, also may be caused by fight attack or labor. Now the emergency treatment and nursing care of them were reported as follows:

\section{Clinical data}

\subsection{Data}

148 cases were included in this study, among which 86 cases were male and 62 cases were female, whose age was between 3-86 years old and average was 45 years old. 72 cases had closed unilateral multiple damage, such as traumatic fracture of multiple ribs, combined with fracture of scapula, fracture of clavicle or hemopneumothorax. 36 cases were open injuries patients, such as bilateral multiple fractures of multiple ribs, fracture of thoracic vertebrae or cerebral contusion. All patients had paradoxical respiration and mediastinal pendular movement. All the patients were received treatment actively, 138 cases were recovered and discharged from hospital but 10 cases died.

\subsection{Treatment methods}

All cases were received corresponding anti-inflammatory, symptomatic and supportive treatment. Thoracic close drainage and chest bandage fixation were received by 148 cases, assisted mechanical ventilator were received by 41 cases, multidisciplinary joint surgery were received by 83 cases. Treatment doctrine: correcting respiratory and circulatory dysfunction, maintaining a smooth breathing and giving oxygen, controlling the external bleeding and supplying blood volume, analgesia, fracture fixation, protecting the spine and so on.

\section{Emergency treatment}

\subsection{Open pneumothorax}

Once diagnosed, patients with open pneumothorax must receive emergency treatment immediately, so that open pneumothorax can be transferred into closed pneumothorax. Emergency treatment measures: a large piece of Vaseline gauze with thick cotton pad or clean clothes are used to cover the wound at the end of a deep breath, and then bind it up so as to close the wound in the chest wall and avoid air leaking. But remember not to plug the clothes in the wound, or maybe cause infection and intrathoracic foreign body. 


\subsection{Tension pneumothorax}

In order to save patients life, syringe needle is inserted into the second intercostal space of the midclavicular line in the injured side to discharge aerothorax, reduce the intrapleural pressure. Moreover, patients should also receive closed thoracic drainage immediately.

\subsection{Flail Chest}

Patients with flail chest caused by multiple fractures of multiple ribs usually also suffer from severe thoracic injury. Therefore, paradoxical respiratory movement should be eliminated or reduced as soon as possible to correct the hyoxemia. Bandage-fixing method, traction and fixation method, operative reduction and internal fixation method, or respirator internal fixation method could be used to correct paradoxical respiratory movement. It is suitable for patients with a wide range of flail chest with severe pulmonary contusion or patients with respiratory distress and hypoxia, which should be assisted ventilation with respirator and to correct intrathoracic abnormal breathing.

\subsection{Hemopneumothorax}

Chest trauma resulting in pleural hemorrhage, called hemothorax. Hemothorax accompanied with pneumothorax called hematopneumothorax. The harm of traumatic hemothorax are acute hemorrhage, pleural cavity forced lung tissue, mediastinal displacement impacting on respiratory function. A small amount of hemothorax (Volume $<50 \mathrm{ml}$ ) could be temporarily observed. Medium dose hemothorax should be supplied blood-volume at first, and then carry out the thoracentesis or closed thoracic drainage at the same time so as to remove the pleural hemorrhage early; progressive hemothorax should receive exploratory thoracotomy timely to stop bleeding; coagulating hemothorax could open the chest to remove the blood clot. Autologous blood transfusion is suitable for the hemothorax within $8 \mathrm{~h}$ after injury, which could recover hemothorax blood by blood recovery system, and then transfuse these blood back to the patients after cleaning and filtering.

\subsection{Cardio great vessels trauma}

Patients with cardio great vessels trauma would suffering massive blood loss, and get into shock in a short period, which could lead to the rapid death of the wounded. For patients with Beck triad (increased venous pressure, blood pressure dropped, low and blunt heart sound) should be alert to the the pericardial blockage. Paracentesis pericardii could be clearly diagnosed. What's more, hematocele could be drawn out to relax the pressure, and to improve hemodynamics significantly, hence to improve the tolerance of anesthesia and surgery. When anti-shock proceeding actively, thoracic surgery should be done against time, to remove hemopericardium rapidly and to repair the damaged blood vessels and heart.

\section{Nursing care}

\subsection{To maintain the airway flow is the most important link in the treatment of severe thoracic trauma}

Because of a variety of multiple trauma factors, there are always sputum and secretions left in the air tube, causing airway blockage, or throat reflex disappeared, or abnormal breathing combined with severe hypoxemia, which should take decisive tracheal intubation, open the airway, timely removal of the nose, blood clots and respiratory secretions, and give sufficient oxygen. For patients with respiratory failure, mechanical ventilation should be used immediately to improve the respiratory function. PEEP ventilation is often used to enhance partial pressure of blood oxgen, hence to ensure tissue oxygen delivery. In this study, 46 patients with respiratory failure were all taken positive and effective mechanical ventilation measures, which had provided the conditions for further rescue.

\subsection{A central venous access was established with the rapid opening of the peripheral veins.}

When opening the peripheral vein, some blood should be reserved as blood specimen hemostasis and other rescue drug. At the same time, central venous access is established. For those gerontal patient with unconsciousness and abnormal heart and pulmonary functions could proceed with anti shock and dilatation treatment under the CVP monitoring. Meanwhile, attention should be paid to master the correct CVP measurement method. For example, patients with ventilator assisted 
ventilation should be measured while offline; or for patients who cannot be offline, measured value must minus the positive end-expiratory pressure. We believe that the femoral vein channel can not be used as a substitute for CVP measurement channel, also venous pressure cannot be measured in PICC catheter.

\subsection{Thoracic closed drainage is the most commonly used treatment for patients with thoracic trauma}

The nursing measures are taken as follows: the patient was lying on the slope; the water-sealed drainage bottle should be placed in the ipsilateral chest level under 60-100 cm of patients; drainage tube should be squeezed usually to maintain drainage patency; to observe closely the fluctuation range of the water column of the drainage tube, and to understand the situation of lung expansion; to observe the quality, quantity, color of the drainage liquid if there were bubbles overflow; to replace the drainage bottles at regular time, aseptic processing was needed strictly; to encourage patients to effectively cough and sputum, deep breathing, changing body position and so on. If hemorrhagic shock appeared after trauma, and still cannot be corrected after a large number of blood transfusion and infusion, and the amount of thoracic cavity drainage is over $200 \mathrm{ml} / \mathrm{h}$ with and last more than $3 \mathrm{~h}$, which could be regarded as progressive hemothorax and be treated timely. If the continuous air leakage is more than $48 \mathrm{~h}$ after the operation, or the X-ray show the atelectasis, which could be diagnosed as bronchus laceration or severe lung tissue laceration. So preoperative preparation should be done timely for the operation. Most of the patients are cured by this method, and some patients are cured after surgery according to the drainage condition.

\subsection{According to the wound site to assist doctors to rescue and preoperative preparation are the guarantee for the success of the rescue}

The patients should check the wound site immediately after admitted to the hospital. If there were knife edge in the patients precordium and the symptoms such as pale complexion, dysphoria, difficult breathing, weak heart stroke, distant heart sounds, low pulse pressure and distension of jugular vein, which could be diagnosed as peicardium tamponade. Patients with such symptoms should be sent to the operation room rapidly and receive the exploratory thoracotomy. For patients with knife edge in the lower part of his chest or downward direction of bullet wound, also with the symptoms such as muscle tension, tenderness, rebound tenderness and dyspnea degree, it is usually thoracic injury combined with diaphragm or inferior organ of diaphragm injury, which should timely report to the doctor and carry out repair operation as soon as possible.

\subsection{Acute respiratory failure is a serious complication of thoracic and cardiac surgery}

For each patients with severe chest trauma, or patients with low blood volume and shock, there is an increase in the volume of dead space ventilation. General anesthesia and intubation, injury pain, and the limitation of breathing machine made insufficient local ventilation, poor expectoration, tracheal secretions increase and stagnation, which may cause pulmonary infection, or even respiratory failure. Therefore, the postoperative patients with severe thoracic injury should adopt the following measures: after fully awake, if the patients had a strong handgrip, the breath machine could be stopped for $30 \mathrm{~min}$. Tracheal cannula could be pulled out and replaced by nasal tube if the patients were checked with blood gas normal; lying at a fowler position if the patients' blood pressure were stable; to assist patients turning over, expectoration and knocking back; when there are some difficult to cough out thick sputum, aerosol inhalation should be done, or aspiration of sputum if necessary; appropriate treatment for pain relief should be given; to control the amount and speed of the infusion. In this study, there was a patients with thoracoabdominal injuries, appeared respiratory failure, breathing and heart stopped after drawing the tube. Later, he was received tracheotomy and success in cardiac massage, but caused lung, diaphragm infection and other complications. So he had to receive the operation again, which was really a profound lesson.

\subsection{Multi parameter monitoring}

Continuous monitoring on heart rate, pulse, blood pressure, blood oxygen saturation, meanwhile observing the respiratory frequency, rhythm, whether there were cyanosis in the endbrush. Under the condition of oxygen inhalation, when the oxyhemoglobin saturation $<0.85$, arterial blood oxygen tension $<60 \mathrm{mmHg}$, breath machine should be used to avoid systemic damage caused by hypoxia. 
Besides, according to the condition of the patients, the ventilation mode and respiratory parameters were adjusted, and giving airway heating, humidifying and nebulization at the same time. For the patients with hemopneumothorax, thoracic close drainage should be done before using the breath machine.

\subsection{Pain and postoperative care}

Chest trauma patients often have chest pain, chest distress, shortness of breath and other subjective symptoms, and often fear of turning over, coughing and deep breathing on account of severe pain, which are easy to cause pressure ulcers, pulmonary infection, atelectasis and other potential complications. Therefore, health education should be carried out to the patients and their families after admission to the hospital. For example, the important significance and methods of turning over and coughing and how to relieve pain. Getting the patient's understanding and cooperation so as to report to the doctor in time when the patient's condition needs to give the pain relief measures. After operation, if the patients' condition is getting better and become conscious, so training such as seat training, standing training can be replaced by walk training gradually. Step one: passive exercise: early exercise is necessary for long-term bedridden patients, while flexion and extension exercise of affected extremity are necessary for physically active patients. The sooner, the better. Step two: from lying down to sitting: From lying to sitting, patients need a process of adaptation. If suddenly sitting, transient cerebral ischemia will be brought out because of the postural changes, and causing pale complexion, dizziness and panidrosis. Therefore, exercise should proceed in an orderly way and step by step. Every day the head of patients need to raise about $10^{\circ}$, more than two times a day, each time $3 \sim 5$ min. After one week, patients can sit well on the bed or chair with a back, and stand on the floor with hand holding up the window lattice tightly. The helper should hold on to patient's shoulder. Exercise should maintain 3 5 times a day, and each time lasting 20 30 min. With the increase in the degree of patients sitting down steadily, helper can gradually withdraw his hand so that the patients can sit down steadily by body balance. Step three: standing exercise: patients cannot stand stable at the beginning, so that helper's assistance is necessary. Standing exercise is lasting 3-5 min each time, and several times a day. Frequency and times can be increased depending on the patients' recovery situation. First, let the patients standing against the wall, assisted by helper whose hands hold up the axillar of patients and both knees stand up to the knee-joints of patients. A few day later, helpers can try to withdraw his hands, and leave patients stand alone against the wall. Therefore, patients can gradually stand alone without help. Next, with the protection of the helper, patients can hold up the bedrails with body doing left and right rotational movement, and further body swinging movement. And then with hands holding up the bedrails, standing up slowing, doing lateral movement, which has laid a basis for walking exercise. Step four: walking exercise: patients hold up to the shoulder of the helper, and helper hold up to the waist of the patients. Marking time is the first step of the walking exercise, walking with slow step. After a few days exercise, walking with crutch for patients can be done without help. The distance of walking with crutch should be from short too long. If the patients want to walk without the crutch, something must be there in the way of exercise as a reliance so that patients can walk steadily alone.

\subsection{Psychosocial Nursing}

Psychological nursing is one of the methods to make the patients to cooperate with the operation and recover soon after operation. Psychological trauma often onset suddenly and fiercely. In the rescue time, almost all patients have the dread feeling with different levels. According to their psychological characteristics, we should explain the medical knowledge related to the disease in a friendly manner and appropriate language, helping them to treat the disease correctly, enhance the courage and confidence to overcome the disease, and also obtaining the trust of patients and their families with a high sense of responsibility and skilled nursing technology. For restlessness patients, restraint strap should be used to restrain the patients (paying attention to the skin in the restraint part). Guardrail should be added to the bed. If necessary, sedative should be injected to the patients according to doctor's advice. Hyperthermia patients $\left(>38.5^{\circ} \mathrm{C}\right)$ should wear icecap, with place ice water bag as pillows, and place ice salt water bag in the bilateral carotid arteries so as to reduce 
cerebral metabolism, relieve cerebral hypoxia, and lessen brain damage. Hospital gown should change in time to prevent a cold. Complicated severe chest trauma is a heave blow for patients and their families, so understanding should be given to the families of the patients, encouraging them to face reality and establishing confidence in the fight against disease. Disease related knowledge should be explained to the patients and their families. Communicating with patients is necessary, and to encourage patients to speak their own ideas.

\section{Reasonable position and environmental factors}

Duo to significantly chest pain of severe chest trauma patients, horizontal position is usually adopted by the patients before the concurrent multiple injuries diagnosed. Semireclining position can be taken appropriately after the exclusion of the chest and lumbar spine fractures. In our hospital emergency department in addition to CT, general inspection require bedside inspection, to reduce movement of the patients so as to prevent pain, blood pressure drop, breathing instability and other risks of patients caused by the change of body position. Therefore, evaluation must be done before moving the patients so as to reduce the risks to a minimum. At the same time, inspection should be done according to the sequence of CRASHPLAN inspection (cardiac-respiration-abdomen-spine-head-pelvis-limbs-artery-nerve). Inspection should be made to maintain airway patency, to supply continuous oxygen to improve brain hypoxia, to clean respiratory secretions in time. When aspiration of sputum, the head of patients should be raised up to 30 degrees with head tilted slightly to one side. Tracheotomy should be made immediately when it is not easy to suck out the sputum. When the head of the patient needs to place the drainage tube, the head of the bed should be pushed into 15-30 degree, connecting the drainage tube under the aseptic operation and hanging it on the bedside, keeping drainage tube unobstructed without and recording the nature and quantity of the drainage fluid. Pulling the drainage tube in the process of movement is strictly prohibited so as to prevent the block of drainage tube. If the drainage tube is blocked by blood clots or sediment, both hands should be used to squeeze the drainage tube until smooth, or aspiration it gently with empty needle under the strict aseptic operation. When going out to check, the drainage tube should be clamped to prevent retrograde infection. Room quiet and the air circulation should be kept, also visitation should be reduced. Close observation of body temperature, pulse, blood pressure, breathing and other vital signs should be given, and also should pay attention to the changes of pupils and consciousness. Make a report to doctor if there are some abnormalities.

Complication prevention: rational use of antibiotics, and to maintain the effective concentration of drugs. Guiding and assisting patients in cough and sputum, excluding respiratory secretions, keeping airway patency and preventing pulmonary complications. In the process of thoracic closed drainage nursing, drainage unobstructed should be kept in strict aseptic operation in order to prevent secondary infection of the chest. Catheter should be placed in a long term, and pay attention to the urethral mouth care to prevent urinary tract infection. Long-term bed ridden patients should pay attention to physical exercise and prevent embolism.

\section{Sound emergency network and effective team assistance are another key to the success of the rescue}

Nursing work must cooperate with the doctor to do the relevant organization, coordination, rescue work. Surgical preparation should be done in a minimum time so that operation could started as soon as possible. 83 cases operation in this group, 6 cases died when receiving multidisciplinary joint surgery. Rescue achievement ratio was $92.7 \%$. High sense of responsibility, acute observing ability, a calm head are necessary during the operation. To ensure that the measures are in place and the operation is accurate. The development of the disease and the flash of many signs requires nurses to have a strong observation and a quick response, so that to win valuable time for the patient's first aid. Meticulous and effective nursing measures have achieved good clinical nursing effect for patients to restore health. Severe thoracic trauma is a common surgical emergency. The thoracic cavity is the 
place for the major organ of the heart, such as the large blood vessels, trachea, lung and so on. Severe chest trauma can lead to respiratory and circulatory dysfunction. Delayed treatment or improper care may cause rapid deterioration of the disease, even life-threatening. Rapid and correct rescue is the key to improve the success rate of severe thoracic trauma.

\section{Conclusion}

In this study, among 148 cases with multiple injuries caused by sever thoracic injury, 138 cases survived, 10 cases died. There were 6 cases died of the 83 cases who underwent by multiple departments at the same time. The emergency treatment and nursing for the multiple injured patients showed that only the nursing staff master the skilled rescuing and nursing care technology, knowledgeable professional theory and comprehensive quality could they deal with the patients timely and accurately. Maintaining breath and blood circulating, observing the state of the illness, strengthening nursing care, preventing from the occurrence of complication played a key role in the sever trauma injured patients.

\section{References}

[1] Weiting Chen. To pay attention and strengthen to the early treatment of severe trauma [J]. Chinese Journal of Trauma. 1999, 15(4):245-246

[2] Lan Zhu. Nursing experience of thoracic injury as multiple injuries [J]. Zhejiang Practical Medicine. 2002, 02(1):7

[3] Kaishi Gu. Thoracic-cardiac operative surgery [M]. Beijing: People's Medical Publishing House. 1985: 407

[4] Yang Huang: First aid and nursing of heart knife wound [J]. Journal of Practical Nursing. 1996, 10: 462

[5] W. Grings. Thoracic injury [M]. Shanghai: Shanghai Translation Publishing Company. 1987: 22

[6] Youning Liu, Liangan Chen, Senyang Yu. Clinical Practice of Mechanical Ventilation (the second edition) [M]. Beijing: Science Publishing Company. 1998, 313: 398. 